\title{
Real Time Stable Haptic Rendering Of 3D Deformable Streaming Surface
}

\author{
Yuan Tian, Chao Li, Xiaohu Guo, Balakrishnan Prabhakaran \\ University of Texas at Dallas \\ 800 W Campbell Rd \\ Richardson, Texas 75080 \\ \{yuan.tian1,chao.li2,xguo,praba\}@utdallas.edu
}

\begin{abstract}
In recent years, many researches are focusing on the haptic interaction with streaming data like RGBD video / point cloud stream captured by commodity depth sensors. Most previous methods use partial streaming data from depth sensors and only investigate haptic rendering of the rigid surface without complex physics simulation. Many virtual reality and tele-immersive applications such as medical training, and art designing require the complete scene and physics simulation. In this paper, we propose a stable haptic rendering method capable of interacting with streaming deformable surface in real-time. Our method applies KinectFusion for real-time reconstruction of real-world object surface instead of incomplete surface. While construction, it simultaneously uses hierarchical shape matching (HSM) method to simulate the surface deformation in haptic-enabled interaction. We have demonstrated how to combine the fusion and physics simulation of deformation together, and proposed a continuous collision detection method based on Truncated Signed Distance Function (TSDF). Furthermore, we propose a fast TSDF warping method to update the deformation to TSDF, and a proxy finding method to find the proxy position. The proposed method is able to simulate the haptic-enabled deformation of the 3D fusion surface. Therefore it provides a novel haptic interaction for virtual reality and 3D tele-immersive applications. Experimental results show that the proposed approach provides stable haptic rendering and fast simulation of $3 \mathrm{D}$ deformable surface.
\end{abstract}

\section{CCS CONCEPTS}

- Computing methodologies $\rightarrow$ Physical simulation; Collision detection; Virtual reality; Reconstruction;

\section{KEYWORDS}

Haptic Rendering, KinectFusion, Deformation, Tele-immersion

\section{ACM Reference format:}

Yuan Tian, Chao Li, Xiaohu Guo, Balakrishnan Prabhakaran. 2017. Real Time Stable Haptic Rendering Of 3D Deformable Streaming Surface. In Proceedings of MMSys'17, Taipei, Taiwan, June 20-23, 2017, 11 pages. DOI: http://dx.doi.org/10.1145/3083187.3083198

Permission to make digital or hard copies of all or part of this work for personal or classroom use is granted without fee provided that copies are not made or distributed for profit or commercial advantage and that copies bear this notice and the full citation on the first page. Copyrights for components of this work owned by others than the author(s) must be honored. Abstracting with credit is permitted. To copy otherwise, or republish, to post on servers or to redistribute to lists, requires prior specific permission and/or a fee. Request permissions from permissions@acm.org.

MMSys'17, Taipei, Taiwan

(C) 2017 Copyright held by the owner/author(s). Publication rights licensed to ACM

978-1-4503-5002-0/17/06 ..\$15.00

DOI: http://dx.doi.org/10.1145/3083187.3083198

\section{INTRODUCTION}

With the development of commodity RGBD cameras like Microsoft Kinects, Apple Primesense, the streaming point cloud is now very easy to acquire to represent the 3D surface. Since the point cloud representation is very natural and beneficial for many aspects such as geometric processing and point-based rendering, it becomes one of the top choices in virtual / augmented reality and tele-immersion applications. Recently haptic rendering of streaming point cloud and RGBD videos have been successfully investigated [4, 14, 19, 21] to incorporate haptic modality to generate more immersive interactions. This emerging research field of "remote touch" naturally inspires the more interesting topics such as: haptic interaction with a streaming deformable surface. Experience of exploring real-world objects are greatly enhanced using haptic feedback. Moreover, treating the explored surface as a deformable model enhances the visual experience during haptic exploration.

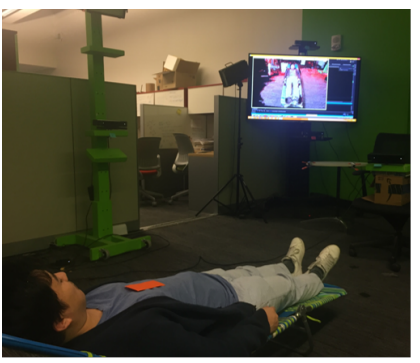

(a)

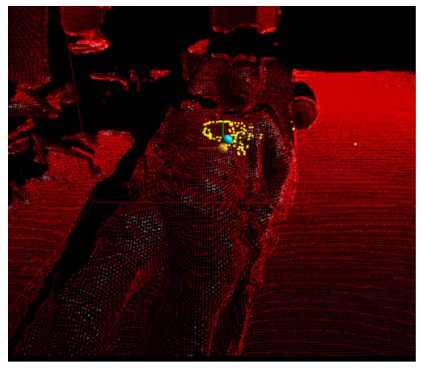

(c)

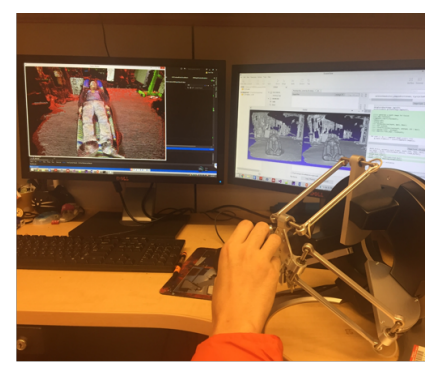

(b)

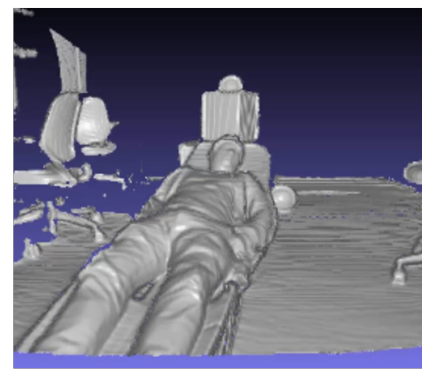

(d)
Figure 1: Wound cleaning tele-immersion training. (a) At local site the trainee uses Kinect to capture the wound, while watching the virtual environment for further instructions. (b) At remote site the physician can use haptic device to interact with the virtual body of the patient. (c) Streaming 3D deformable surface of patient. (d) TSDF. 
Many real-time tele-immersive applications such as remote emergent training, art designing and collaborative works need more flexible and interactive ways of haptic interaction, including complex physics simulation such as deformation. We have implemented the wound cleaning tele-immersive training system using our method, as shown in Fig. 1. The wound cleaning training use the Kinect for the patient to capture the wound, and remote physician can use haptic device to interact with the point cloud to show the trainee where and how to clean the wound. Finally, the trainee at the local site can follow the instructions to clean the wound of the patient in real-world. Handling streaming point cloud as surface is itself a challenge since the data is noisy and incomplete to reflect the real dense geometric features. The other challenge is the stability of collision detection between point cloud and haptic interface point (HIP). The failure of collision detection will lead to the popthrough phenomenon, causing HIP to penetrate through the surface point cloud. Third challenge is to meet the requirements of high update rate for haptic device and ensure smooth haptic and visual rendering. The fourth and final challenge is the simulation of the deformable surface continuously. The deformation of surface needs to be natural and in a continuous manner even if the camera is moved on the fly to explore the scene in detail. The movement of camera is very natural phenomenon in many tele-immersive applications as it allows to explore the real-world scene and to capture the details of an object from different angles.

In this paper, we have proposed a method for real-time haptic rendering of the streaming deformable 3D surface. The proposed method applies the real-time KinectFusion [12] to fuse the streaming frames of the static scene, where the fused Truncated Signed Distance Function (TSDF) is naturally a smooth surface representation. A continuous ray-casting collision detection method is proposed to avoid the missing or failed collisions, and a fast proxy update way is proposed for haptic rendering. This method applied the hierarchical shape matching method (HSM) similar to [25], which generates the fast and stable simulation of the deformable meshless surface. A fast TSDF warping after deformation is introduced for fast update the warped TSDF after deformation. Furthermore, this method builds the deformation field with the same size of the TSDF volume, therefore the $3 \mathrm{D}$ deformation is simulated continuously while fusing. Finally all processing intensive operations such as fusion, collision detection, and simulation are parallelized using GPU, which provides the real-time efficiency.

Specifically, the technical contribution of this work can be summarized as follows:

- We present an efficient and robust method that allows a realtime haptic interaction with 3D deformable streaming surface which is under reconstruction. The deformation inherits the unconditional stability and high efficiency of HSM approach, the deformation field can simulate the 3D deformation continuously while performing KinectFusion.

- A fast TSDF warping algorithm is proposed to efficiently update the warped TSDF after deformation.

- A real-time TSDF based continuous collision detection method is proposed to minimize missing detection of collisions, thereby enhancing haptic interaction experience.

- An efficient proxy finding algorithm is proposed to find the proxy in TSDF volume for proxy-based haptic rendering.

\section{RELATED WORK}

This section is divided into three parts to introduce the related work of different fields.

\subsection{Haptic Interaction with Streaming Data}

In recent years, the streaming RGBD / point cloud data has gained growing popularity in haptic rendering field. Haptic rendering methods with the depth image and the RGBD video are first introduced in $[4,5]$, which apply tessellation to represent the surface for each depth frame offline, and then use proxy-based method to compute the force. Implicit function is the other popular way to represent the 3D surface [22], recently it has been used for hapticenabled interaction. Guo proposed implicit representation for static point set which supports for haptic-enabled deformation [10, 11]. Leeper proposed two implicit function to describe the surface of streaming point cloud [14], and then exploited the 3-DOF (degrees of freedom) haptic rendering method on the implicit surface. Ryden [21] applied the similar surface representation which is the neighboring small surface patch near HIP, and used an adaptation proxy method [20] by iteratively moving proxy toward HIP while estimating the surface normals. The representation of streaming surface is quite challenging, therefore most previous work use smoothing / weighted averaging to estimate the surface. One evident problem of the previous work is the incomplete streaming data, as most realistic scenes cannot be fully captured by the single sensor. Leeper [14] used multiple calibrated cams to alleviate the problem. In our method, we applied an alternative way to represent the surface using KinectFusion [11], that fuses in real-time all the streaming data captured through a Kinect sensor into a global implicit surface model (TSDF). KinectFusion can be flexibly used for the full-scene capture to enhance the haptic interaction, and provide a smooth implicit surface. Another problem is that real-time physics of the point cloud has not been investigated in this field. Therefore, we proposed the method to simulate deformation of the streaming surface, which is the most common physical phenomenon in the real world.

\subsection{Collision Detection in Haptic Interaction}

Previous haptic rendering methods with streaming data use bounding volume hierarchy or Kd-tree $[14,21]$ to speed up the collision detection, however the detection is likely to fail due to the noisy change of local surface when HIP is moved fast by the user. In previous work [21], the collision detection method is to project the bounding volume of HIP to depth image to find nearest points, and directly check whether there is intersection between the HIP and nearest points at discrete time step. Leeper [14] applied a similar method and used Kd-tree to speed up the detection. After finding the potential region of point cloud, Leeper used this region to generate the implicit surface which will easily tell whether HIP is inside or outside the surface. To our best knowledge, previous methods mostly applied discrete collision detection by considering the HIP position and related surface normal, which will easily lead to the HIP pop-through phenomenon for the haptic interaction. Compared with discrete collision detection, continuous collision detection $[2,3,23]$ provided more robustness by detecting in the 


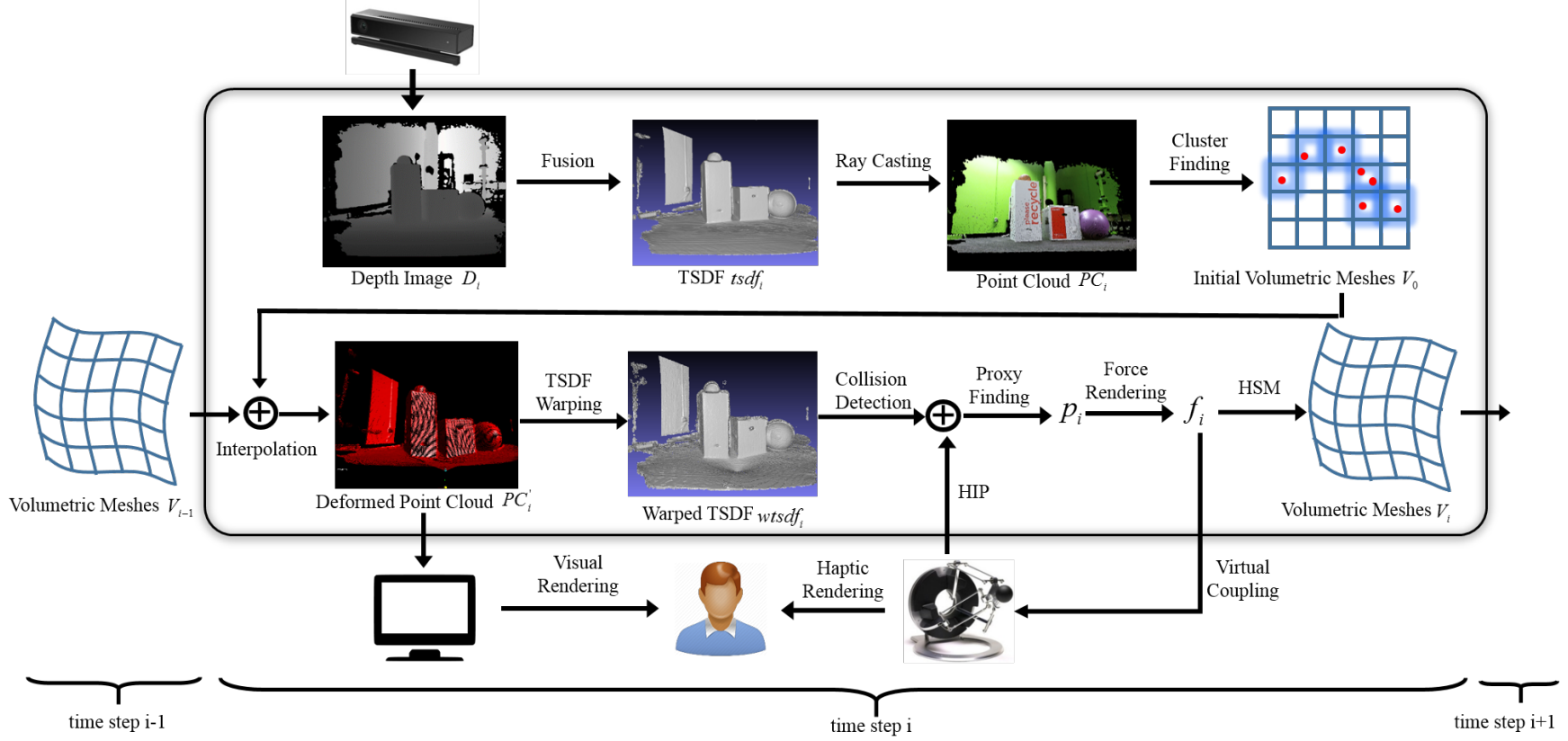

Figure 2: The pipeline of the proposed method.

time period between two discrete time steps. Recently more stable continuous collision detection method [27] is introduced for haptic rendering with $3 \mathrm{D}$ objects. To avoid the pop-through failing detection, we have proposed a continuous collision detection using TSDF and raycasting.

\subsection{Haptic-enabled Deformation}

Enabling the simulation of the deformable surface with the haptic rendering has been an active research area. Many methods are proposed for realistic rendering of interacting static / deformable 3D surface mesh, volumetric image [6, 7, 13]. Haptic-enabled large deformation of soft objects was proposed in [9]. Barbic [1] proposed an efficient 6 DOF simulator between the rigid-body object and reduced FEM deformable object, and used penalty-based method for haptic rendering. Tian [24] applied an alternative meshless shape matching method [15] for haptic-enabled fast simulation of soft objects. Later this method is extended to adaptive hierarchical shape matching (HSM) to achieve large deformation and stable haptic rendering [25]. Our approach has applied the similar idea, since this method is compatible with all kinds of surface representation, computationally fast and stable.

\section{OVERVIEW}

The proposed method introduces real-time haptic rendering of 3D deformable object under reconstruction. It applies KinectFusion to generate TSDF implicit representation for the streaming surface [17]. In the fusion process, each streaming depth data is first converted into 3D points, and Iterative Closest Point (ICP) is applied to track the camera. Given the global pose of camera, a reference 3D TSDF volume is updated that each voxel stores a running average of distance value. TSDF suits our requirements because of its advantages with haptic interactions: It implicitly smoothes the depth data noise resulting in the stable haptic rendering.

In our method, the surface geometry will be rendered as point cloud which is deformed using the hierarchical shape matching (HSM) [25]. The hierarchy structure is able to speedup the deformation propagation. In this work, the hierarchical volumetric meshes are initialized using different resolution: the top-level volumetric mesh is generated by voxelizing the bounding volume (cube) with the same size of the reference TSDF volume, and each voxel is divided for next level using Octree data structure. The vertex of the voxel and all its neighboring connected vertices form one "cluster", and each vertex of the voxel represents one node of corresponding cluster.

The deformation energy of each cluster $E$ is defined as the square distance between current positions and the goal positions after linear transformation:

$$
E=\sum_{i} m_{i}\left\|\mathbf{R} \mathbf{x}_{i}^{0}+\mathbf{t}-\mathbf{x}_{i}\right\|^{2} .
$$

where $\mathbf{R}$ and $\mathbf{t}$ are rotation and translation for one cluster, $\mathbf{x}_{i}^{0}$ and $\mathbf{x}_{i}$ are the initial position and goal position of each node, $m_{i}$ is the mass for one node. This optimization problem can be solved by the polar decomposition, which is performed independently for each cluster. Therefore it is very suitable for parallel computing using GPU.

Our method uses the multi-rate architecture [18] splitting the haptic rendering pipeline into a haptic thread and a simulation thread. is currently fused by KinectFusion which is only for generating 3D point cloud. At any time, our method maintains two TSDFs in the memory. One is $t s d f_{i}$ that is currently fused by KinectFusion and used only for generating 3D point cloud. Another is $w t s d f_{i}$ that saves the implicit surface after deformation. Note that, $w t s d f_{i}$ is used by the collision detection and proxy finding modules. 
The pipeline of our method is described as shown in Fig. 2, the main steps are summarized as follows:

1) For current frame $i$, the system captures Kinect depth image remotely, and then performs the fusion, update current $t s d f_{i}$. The current point cloud is generated through raycasting. This fusion step is exactly the same as [17].

2) The corresponding clusters in initial hierarchical volumetric meshes are found for each point in the generated point cloud. The deformed volumetric meshes $V_{i-1}$ from previous $i-1$ frame is used to interpolate the deformed physical surface point cloud.

3) The warped TSDF $w t s d f_{i}$ is generated through TSDF warping.

4) The HIP position is captured after warping, and $w t s d f_{i}$ is used to perform continuous collision detection.

5) Since proxy-based method is applied [20], the proxy of HIP is found if there is collision by our proxy finding algorithm.

6) Based on the external haptic force computed at previous step, hierarchical shape matching (HSM) is performed for volumetric meshes deformation.

The haptic thread is very straightforward in that it gets the spring force by proxy-based rendering, and performs virtual coupling to render the force to haptic device.

The following paragraphs describe more details of the proposed method. Sec.4 describes how we update the deformation for the newly fused surface, Sec.5 describes our TSDF warping algorithm, Sec.6 introduces our continuous collison detection, finally Sec.7 describe the proxy finding algorithm in TSDF space.

\section{SURFACE DEFORMATION UPDATE}

In this section, we will describe how to update the surface deformation. Updating the surface deformation on the fly a non-trivial step, since KinectFusion will fuse more geometry for the 3D surface, and simulation of deformation will change the 3D surface simultaneously. Because, when the surface is changed through the simulation of the deformation, it is also updated by the KinectFusion to fuse more 3D surface geometry.

To perform hierarchical shape matching (HSM), Hierarchical volumetric meshes with different resolution are built with the same volume size of TSDF, as shown in Fig. 3. These volumetric meshes will perform physics simulation instead of direct deformation of point cloud. There are two advantages to perform simulation in such way: firstly volumetric meshes are built with the same size of TSDF volume, when large 3D scene is reconstructed, the simulation field will always cover the scene. Secondly, since the volumetric meshes are deformed instead of the point cloud itself, the deformation will be continuous and smooth, it will not be influenced by KinectFusion process, and vice versa. The point cloud including the newly added region by KinectFusion will be updated by the deformed volumetric meshes.

After the KinectFusion step in the Fig. 2, a new point cloud is generated by the newly fused TSDF. To render the visual result of deformation, this point cloud needs to be updated based on the deformation simulation of previous time step. In HSM, after the one time-step deformation of the volumetric meshes, the bottom level will interpolate the geometric model. In our method, the point cloud is newly created because of fusion at each time step, therefore the correspondence between bottom-level volumetric mesh and

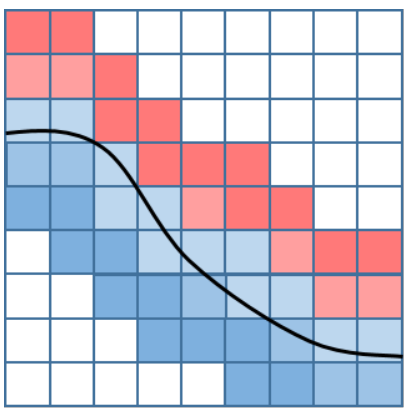

(a)

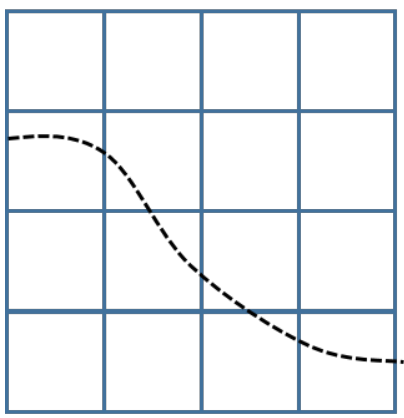

(c)

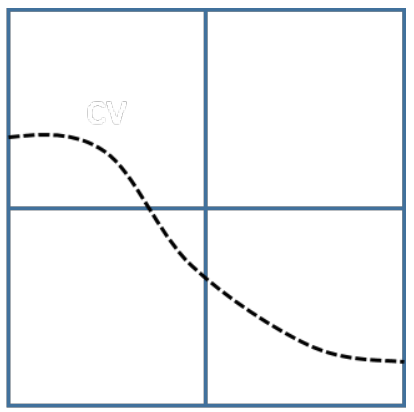

(b)

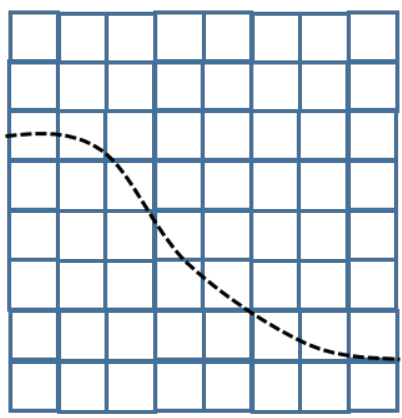

(d)
Figure 3: Surface modeling based on HSM, all hierarchical volumetric meshes have the same size of TSDF volume. (a) TSDF. (b) Volumetric mesh on first level. (c) Volumetric mesh on second level. (d) Volumetric mesh on third level.

point cloud will be found before interpolation. In other words, each point needs to find related clusters.

In our method, 4 nearest neighboring clusters of each point are found in the rest bottom-level volumetric mesh $V$. Each neighboring cluster is named as an active cluster. The interpolation weight will be computed for this corresponding point as:

$$
w_{i}(\mathbf{x})=e^{-\| \mathbf{v}_{i}-\mathbf{x}||^{2} /\left(2 \sigma_{i}^{2}\right)}
$$

where $w_{i}$ is the interpolation weight for $i$ th cluster, $\mathbf{x}$ is the point position, $\mathbf{v}_{i}$ is the centroid position of cluster $i$ in rest volumetric mesh, and $\sigma_{i}$ is a radial basis parameter to describe the cluster influence radius. Each interpolation weight will be further normalized:

$$
w_{i}^{\prime}(\mathbf{x})=\frac{w_{i}(\mathbf{x})}{\sum_{i} w_{i}(\mathbf{x})}
$$

The new deformed position of the point $\mathbf{x}$ is interpolated as follows:

$$
\tilde{\mathbf{x}}=\sum_{i} w_{i}(\mathbf{x}) \tilde{\mathbf{v}}_{i}
$$

where $\tilde{\mathbf{v}}_{i}$ is the cluster centroid in the deformed bottom-level volumetric mesh. In HSM, the bottom level has fine resolution therefore bottom-level volumetric mesh is used for interpolation.

Our active cluster finding algorithm is very fast because it can be implemented in GPU and has $O(1)$ time complexity for each point without any search. As shown in Alg. 1, it uses one round 
float operation to find the nearest cluster index for the point, and then based on the vector from the cluster centroid to the point, it finds the other 3 nearest clusters.

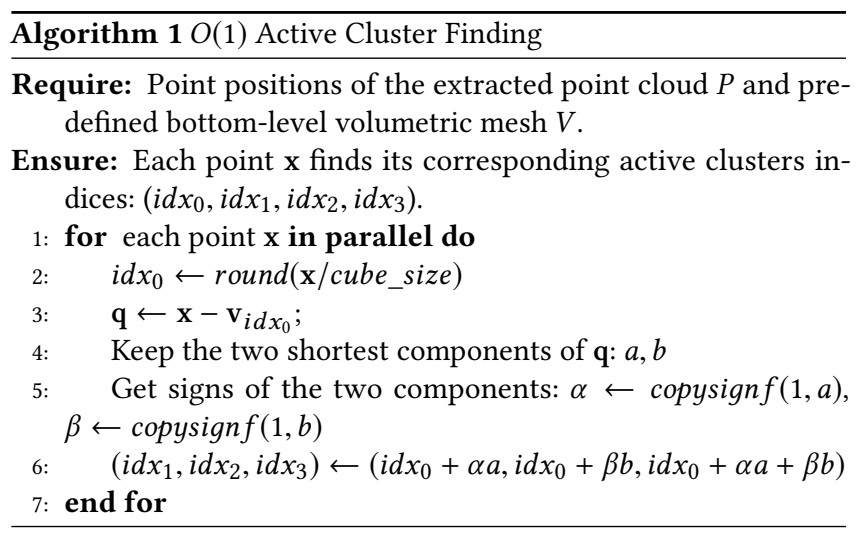

After the cluster finding step, the deformed volumetric mesh from previous time step will interpolate the deformed surface point cloud, as shown in Fig. 2.

\section{TSDF WARPING}

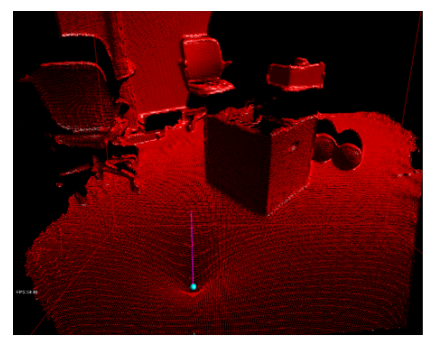

(a)

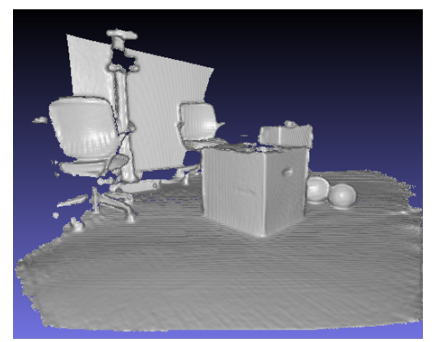

(c)

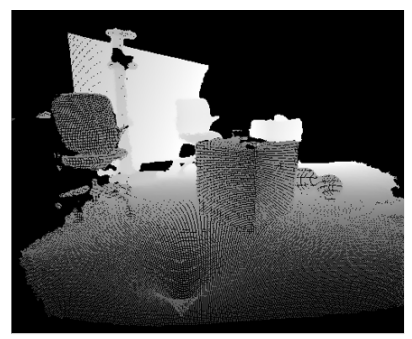

(b)

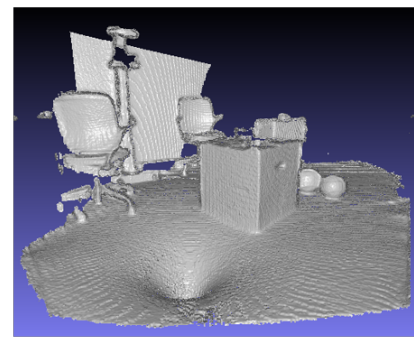

(d)
Figure 4: TSDF warping. (a) current deformed point cloud, the sphere is the haptic tool, and purple segment represents the force vector. (b) projected depth image by point cloud. (c) warped TSDF in previous time step. (d) current updated warped TSDF.

The warped TSDF $w t s d f$ is initialized as the regular TSDF at the first frame. After the deformed surface is generated, the proposed method will update the deformation in the warped TSDF, which will be utilized to perform the following collision detection and handling.
In previous work, Vaillant [26] proposed a distance field reparameterization method for skinning animation which uses Hermite Radial Basis Functions to approximate each bone segment and solves an optimization problem. This algorithm needs skeleton bones and skinning weights as preliminary knowledge but performs very well for skinning animation. Fisher and Lin [8] computed partial distance field to accelerate the update of the deformed distance field for a polygon or polyhedron mesh.

Compared to methods mentioned above, our system aims to achieve the high update rates for both visual rendering and haptic rendering, therefore we made two assumptions for the deformation: Since our deformation is based on the volumetric meshes instead of polygon mesh, and in our system, users are always tracking the deformed region on the screen, it is assumed that the deformation mainly happens in the current view which has the same view angle as the Kinect. Another assumption we make is that the haptic-enabled deformation is not as large as the one for skinning animation. In our scenarios, severe occlusions that often occur in skinning animation due to large deformation seldom happen.

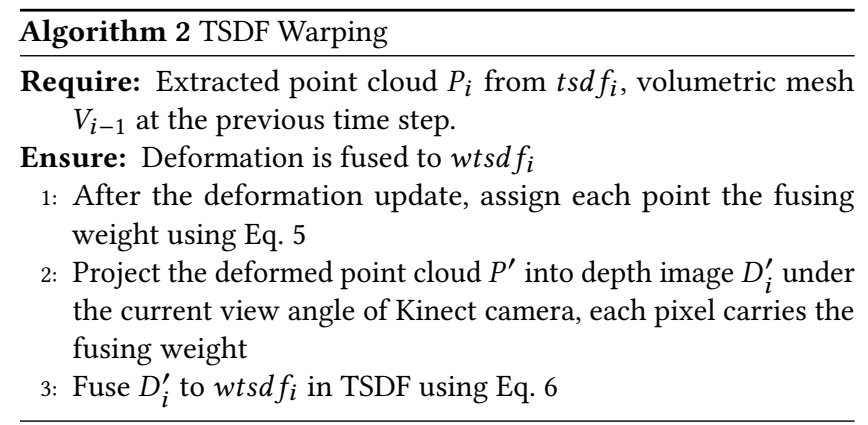

After updating surface deformation as described in Sec. 4, a deformed point cloud $P_{i}^{\prime}$ is generated by the previous volumetric mesh $V_{i-1}$. The straightforward way to update the warped TSDF is to find the correspondence between the voxels in TSDF and the deformed point cloud using closest-point search method. However, this method is time consuming and cannot guarantee the high update rate. In our work, we propose a TSDF warping method that can meet our requirments very well. It is not only updated very fast on GPU, but also fits our entire framework and raycasting based collision detection.

Based on the two assumptions of our method, the deformation is not large and surely in current camera view, we can borrow the fusion idea to update the warped TSDF. Similar to the process of raycasting rendering, the point cloud $P_{i}^{\prime}$ is projected onto a $2 \mathrm{D}$ depth image $D_{i}^{\prime}$ corresponding to the current camera angle, as shown in Fig. 4(b). Finally, similar to KinectFusion, depth image $D_{i}^{\prime}$ is integrated into the warped TSDF $w t s d f_{i}$, as shown in Fig. 4(c)(d). Different from the regular KinectFusion method, our method computed the fusion weight based on the deformation energy. The goal of such fusion weight is to quickly update the deformation region of the surface. If one region has zero deformation energy, then this region should perform the regular volumetric integration in warped TSDF. If one region has some deformation, then this region needs to be updated quickly instead of regular weighted smoothing. 
Therefore, we define the fusion weight as shown in Eq. 5:

$$
\alpha_{i}=e^{\sum_{j} w_{j} E_{j}}
$$

where $\alpha_{i}$ is the fusion weight for one point at $i$ th time step,to simplify the representation, the index of point is omitted. $w_{j}$ is the interpolation weight described in Eq. $2, E_{j}$ is the $j$ th cluster deformation energy. When there is no deformation on the surface, the fusing weight value equals 1 and will guarantee the regular TSDF integration described in [17]. This fusing weight will be carried for each pixel in $D_{i}^{\prime}$, so that it will not miss any deformation details: the larger energy the corresponding point has, the larger weight the voxel has when fused to $w t s d f$.

After the weight assigning, the volumetric integration can be described as Eq. 6 :

$$
\begin{aligned}
\beta_{i} & =\min \left(\text { WEIGHT_MAX, } \alpha_{i}\right) \\
w t s d f_{a v g} & =\frac{w t s d f_{i-1} \beta_{i-1}+w t s d f_{i} \beta_{i}}{\beta_{i-1}+\beta_{i}}
\end{aligned}
$$

Where $\beta_{i}$ is the current fusing weight at $i$ th time step, WEIGHT_MAX is the max value of weight, which is usually set as 64 . If the weight is too large, fusion will have a lag when deformation recovers (when deformation energy decreases), WEIGHT_MAX is a threshold heuristically selected to cut off too large weight. wtsd $f_{a v g}$ is the final updated value, $w t s d f_{i-1}$ is the TSDF value at last time step, $w t s d f_{i}$ is the input TSDF value estimated from the depth image. The integration algorithm is following the KinectFusion, can run in parallel using GPU.

The whole working frame of TSDF warping is described in Alg. 2.

\section{TSDF-BASED CONTINUOUS COLLISION DETECTION}

Most previous work of haptic interaction with streaming point cloud [14, 21] applied discrete collision detection. However, in such applications, the HIP is moved arbitrarily by the user and its moving speed is very high, so the detection may fail and HIP may pop through the point cloud. To overcome this drawback, in this section, we introduce the TSDF-based continuous collision detection method.

KinectFusion uses fast raycasting [17] onto the estimating depth frame to provide a dense surface, which has inspired our TSDFbased collision detection method. The HIP position is denoted as $\mathbf{h}_{i}$ at $i$ th frame, which can be transformed to the reference frame of TSDF volume: $\mathbf{h}_{i}^{\prime}=\mathbf{T}_{g, k} \mathbf{h}_{i}$, where $\mathbf{T}_{g, k}$ is the camera pose estimation for a frame at time $k$. The signed distance value of HIP position is looked up as $w t s d f\left(\mathbf{h}_{i}^{\prime}\right)$.

Recall the raycasting for surface reconstruction in Kinectfusion: one pixel's ray starts marching from the minimum depth and stopping when a zero crossing (+ value to - value for a visible surface) is found as the surface interface. Therefore, collision detection becomes the problem of raycasting from $\mathbf{h}_{i-1}^{\prime}$ to $\mathbf{h}_{i}^{\prime}$ and find whether there is the surface interface in TSDF volume. In other words, the detection is to find if there is an intersection between the surface and line segment $\overline{\mathbf{h}_{i-1}^{\prime} \mathbf{h}_{i}^{\prime}}$. Following this idea, two types of collision detection are proposed:

First contact collision detection is to detect the first collision between the surface and HIP. In the system configuration, the haptic device is initialized as outside of the surface which means the HIP

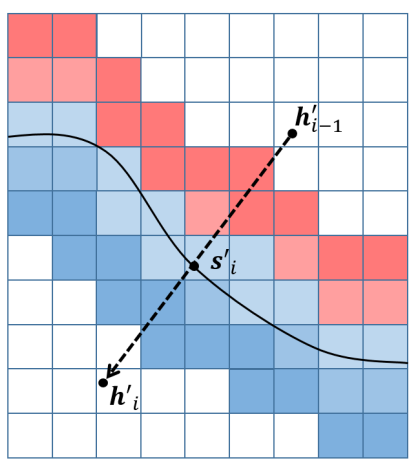

(a)

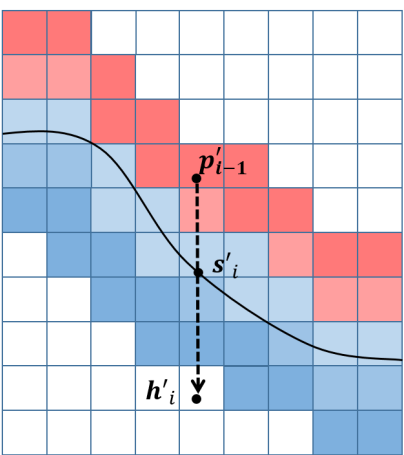

(b)
Figure 5: TSDF-based continuous collision detection in 2D scenario, where $\mathbf{h}_{i}^{\prime}, \mathbf{p}_{i}^{\prime}$, $\mathbf{s}_{i}^{\prime}$ represent $i$ th frame's HIP, proxy, and intersection separately. The curve is the physical surface, voxels represent the TSDF volume. Voxels with color are truncated signed distance fields, red voxels are exterior voxels (+ value) and blue voxels are interior ones (- value). (a) First contact collision detection. (b) Subsequent contact collision detection.

signed distance value is bigger than 0 . Therefore at $i$ th frame, a ray is built in the TSDF reference frame from $\mathbf{h}_{i-1}^{\prime}$ to $\mathbf{h}_{i}^{\prime}$, as shown in Fig. 5(a). Similar to fusion, the raycasting is performed from the minimum depth to the maximum to find if there is zero crossing changing from + value to - value. If collision happens, the surface intersection position $\mathbf{s}_{i}^{\prime}$ is set to be the surface interface.

Subsequent contact collision detection happens after that HIP is inside the surface. Since the proxy-based method [20] is used for haptic rendering, a proxy $\mathbf{p}_{i}$ will be rendered on the surface, which can be transformed to TSDF frame: $\mathbf{p}_{i}^{\prime}=\mathbf{T}_{g, k} \mathbf{p}_{i}$. As shown in Fig. 5(b), due to the surface deformation at $i$ th time step, the previous proxy position $\mathbf{p}_{i-1}^{\prime}$ is now outside of the surface instead of on the surface. To detect the subsequent contact on the surface, a ray is built from $\mathbf{p}_{i-1}^{\prime}$ to $\mathbf{h}_{i}^{\prime}$, which is from last time-step proxy to current HIP. The raycasting process is the same with first contact collision detection. The intersection position is found as $\mathbf{s}_{i}^{\prime}$ if collision happens, which is used to find the proxy position. If there is no collision, then HIP is now getting out of the surface, current proxy position will be set as HIP position.

The proposed TSDF-based collision detection is a standard continuous collision detection, since it finds the exact time between frame $i-1$ and frame $i$ when crossing the zero isosurface in the distance field. This method will avoid the pop-through of fast HIP movement, therefore provides collision stability.

\section{PROXY FINDING}

To generate the collision response after the collision happens, we applied the proxy-based haptic rendering [20]. The proxy is defined as the HIP's nearest surface point $\mathbf{p}_{i}^{\prime}$ in the warped TSDF reference frame. It is found by minimizing $\left\|\mathbf{p}_{i}^{\prime}-\mathbf{h}_{i}^{\prime}\right\|$ subject to the constraint $w t s d f_{i}\left(\mathbf{p}_{i}^{\prime}\right)=0$.

For implicit surface, this problem can be solved by iteratively moving the HIP towards the approximated gradient direction to 
approach the closest surface point [22]. In signed distance function field, it is much easier since each point inside will get the accurate gradient. However, since the distance values in TSDF are truncated, and gradients of points which are outside of truncated region are hard to estimate. Fortunately, it is observed that the great advantage of TSDF representation is its natural support for very fast and convenient raycasting. Therefore we proposed a raycasting way to lookup the gradient of one point in TSDF volume if signed distance value is not available.

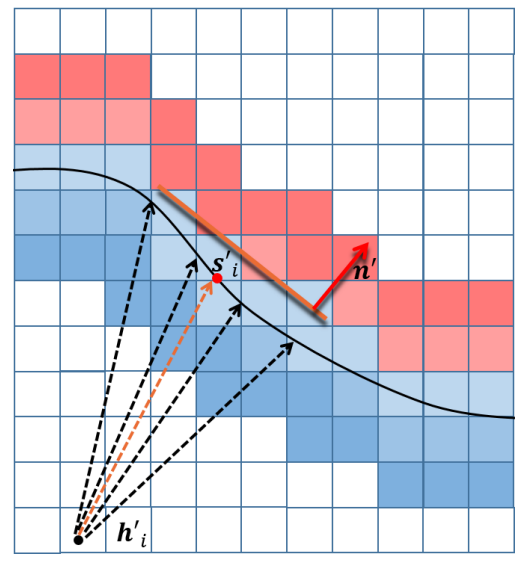

Figure 6: Raycasting to find gradient of TSDF. $h_{i}^{\prime}$ is the HIP point, $s_{i}^{\prime}$ is the intersection surface point returned by the TSDF collision detection phase. A number of rays are sampled in spherical coordinates frame based on the direction from $h_{i}^{\prime}$ to $s_{i}^{\prime}$.

As shown in Fig. 6, given the current HIP position $\mathbf{h}_{\mathbf{i}}^{\prime}$ and surface intersection position $\mathbf{s}_{\mathbf{i}}^{\prime}$, lots of rays are generated for raycasting starting from the HIP. The ray directions are uniformly sampled in spherical coordinates from the neighborhood of the direction from HIP to intersection position. After raycasting, the intersection points are generated as a point set, then the average weighted normal is computed as the gradient of TSDF at HIP position (to simplify, the variable is denoted without frame subscript $i$ ):

$$
\nabla w t s d f\left(\mathbf{h}^{\prime}\right) \approx \mathbf{n}^{\prime}=\left(\Sigma_{k} \omega_{k} \mathbf{n}_{\mathbf{k}}^{\prime}\right) /\left\|\Sigma_{k} \omega_{k} \mathbf{n}_{\mathbf{k}}^{\prime}\right\|
$$

where $\omega_{k}=1.0 /\left(\mathbf{n}_{\mathbf{k}}^{\prime} \cdot\left(\mathbf{s}_{\mathbf{k}}^{\prime}-\mathbf{h}^{\prime}\right)\right)$. After getting the normal, raycasting from HIP along the normal direction will find the nearest surface interaction point $\mathbf{s}^{\prime}$. Therefore TSDF value is estimated as:

$$
w \operatorname{tsd} f\left(\mathbf{h}^{\prime}\right) \approx \mathbf{n}^{\prime} \cdot\left(\mathbf{h}^{\prime}-\mathbf{s}^{\prime}\right)
$$

Since raycasting can estimate both gradient and signed distance value, the proxy finding algorithm is proposed similar as the optimization in [22], as shown in Alg. 3. Starting from the HIP position, the approximated position of proxy will move forward along the gradient direction, finally it will arrive the optimal position. The proxy finding process is executed in GPU therefore it is very efficient. Fig. 7 shows the proxy finding procedure.

After the proxy finding, a spring force will be computed as $\mathbf{f}=-k\left(\mathbf{p}^{\prime}-\mathbf{h}^{\prime}\right)$ and this force will be transformed from TSDF coordinates to the camera coordinates. The haptic thread will take this force and add virtual coupling to generate a stable force for haptic

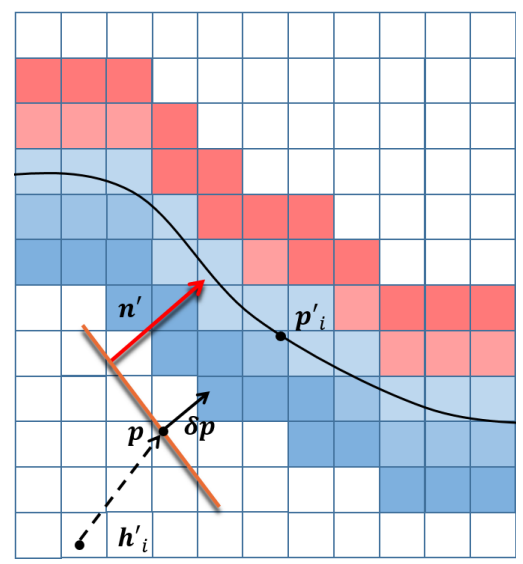

Figure 7: Proxy finding in warped TSDF. $h_{i}^{\prime}$ is the HIP point, $p$ is the approximated point starting from HIP point, $p_{i}^{\prime}$ is the unknown optimal proxy position. Each optimization iteration, $\mathrm{p}$ will be moved by $\delta \mathrm{p}$ along the gradient direction.

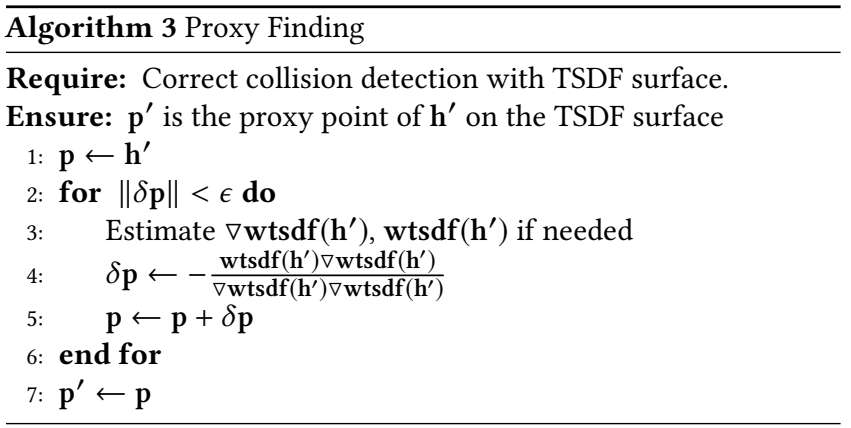

device. Meanwhile, the spring force is applied for the neighboring shape matching cluters, using Alg. 1 . The deformable simulation then begins with the shape matching iteration from the top level to the bottom level. The upper level clusters ( $R$ and $\mathbf{t}$ ) will be set as initial rotation and translation of lower-level clusters. The algorithm is shown as Alg. 3.

\section{EXPERIMENT RESULTS}

In this section, the experiment results are shown to demonstrate the performance of the proposed method, please also refer to the demo video for more details of the experiments: https://youtu. be/k8ddXPiwiCo

All experiments were performed on Intel i7 $3.50 \mathrm{GHZ}$ machine with 32 GB RAM, the GPU is GeForce GTX 670. For hierarchical shape matching, we apply 3 -level hierarchy with $8^{3} / 16^{3} / 32^{3}$ clusters for each level. The stiffness for the surface is easy to adjust in HSM. In KinectFusion, the dimension of the TSDF volume is set to be $512^{*} 512^{*} 512$, voxel size is about $10 \mathrm{~mm}$, the truncated depth distance is set to be $50 \mathrm{~mm}$. For camera tracking 3-level ICP structure is applied, the ICP iterations of levels are $4 / 5 / 15$, which is efficient for indoor tracking. 


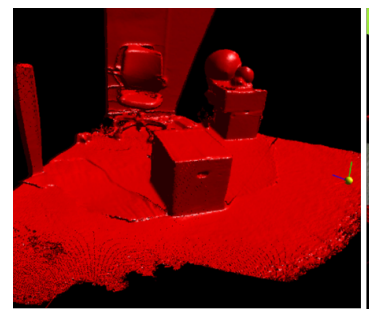

(a)

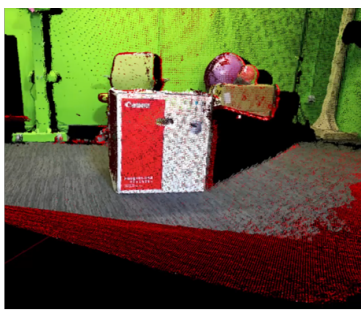

(b)

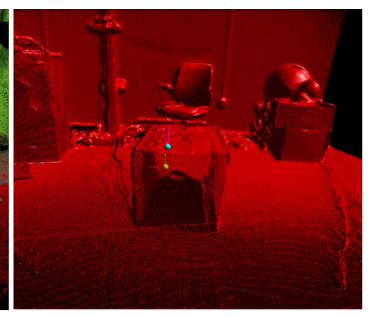

(c)

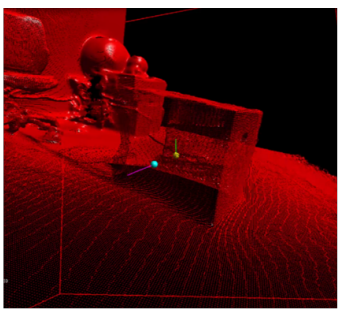

(d)

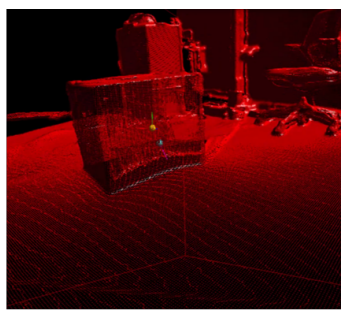

(e)

Figure 8: An example of our method that uses haptic device to interact with four surfaces of the box while 3D fusion. The blue sphere is the proxy, yellow sphere is the HIP, magenta segment is the force vector. (a) Reconstructed 3D box. (b) Current captured streaming point cloud. (c) Haptic interaction with the top face. (d) Haptic interaction with the left face. (e) Haptic interaction with the right face.

\subsection{Deformation and Fusion}

Comparing with the previous work [4, 14, 21], the evident advantage of our method is the real-time 3D reconstruction by KinectFusion. Most of the previous methods utilized a single depth camera or the static configuration of the multiple depth cameras to capture the scene, which limits the scenarios of potential applications of the system. In our method, one user can use Kinect to scan any region of interest in indoor scenes quickly while the other user can use haptic device to interact with the streaming surface simultaneously. Fig. 8 shows one example to demonstrate the flexibility by the fused 3D surface. In this example, a box is reconstructed as an object of interest, current camera view only shows the front of the box in Fig. 8(b). Since all faces of box are already scanned and fused, our method enables the user to interact with all the faces of the 3D box regardless of the current camera angle. Some methods [14] support for multiple calibrated cameras, however these cameras need to stay in place, there is still the problem of visual occlusion. In contrast, the proposed method can provide the users more flexible ways to capture the indoor scenes.

The proposed method has clearly separated the physical simulations and 3D reconstruction steps. Hence, it is possible to achieve temporally smooth deformation of the 3D surface even if the deformed region has new fused points. We have made an experiment to demonstrate how deformation is smooth at the fusing region. As shown in Fig. 9, HIP is firstly moved to the position that deforms the boundary of point cloud. HIP is then kept still, and Kinect is moved towards the boundary region to fuse more geometry. During this procedure, the newly fused surface region is smoothly deformed and well integrated into the existing surface.

\subsection{TSDF Warping}

We have proposed the TSDF warping method to update deformation to the warped TSDF. The TSDF warping firstly compute the fusion weight for each point from its nearby cluster deformation energy. The weights are carried by the projected depth image, and used to generate the new TSDF value. We have compared the TSDF warping with and without such weights, the results are shown in Fig. 10. The results demonstrate that the fusion weight can fast update the deformed region.

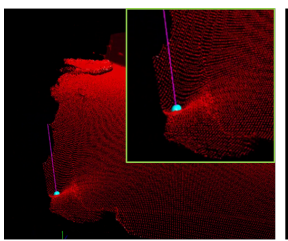

(a)

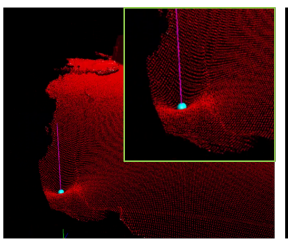

(b)

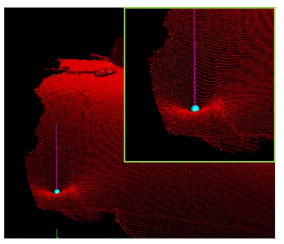

Figure 9: The deformation of the surface region that is being fused. $(a)(b)(c)$ are the deformation results at 3 consecutive time steps. In this process, HIP is not moved but Kinect camera is moved to fuse more surface details. The deformation in this process shows great smoothness.

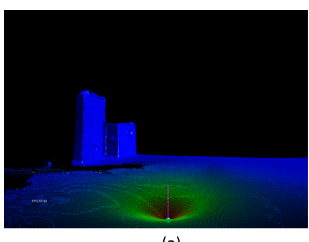

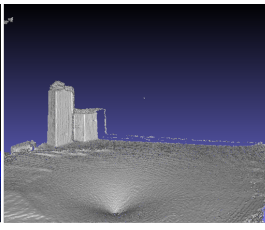

(b)

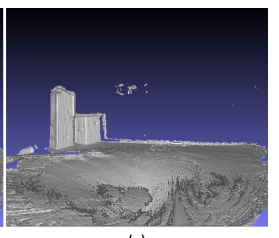

(c)
Figure 10: The TSDF warping results with/without fusion weights. (a) The deformed point cloud, which is rendered based on the weight distribution, the colors from blue to red corresponds to the weights from small to big. (b) Current warped TSDF that is updated with fusion weight. (c) Current warped TSDF that is updated without fusion weight.

\subsection{Collision Detection}

The proposed TSDF-based continuous collision detection facilitates to avoid the HIP pop-through. To demonstrate the effectiveness of the proposed method, we compare it's performance with the methods proposed in $[14,21]$ which are discrete deformation methods. These methods used Kd-tree / bounding volume hierarchy to quickly find the potential nearby region of HIP at each time step, and utilized the identified region as an implicit plane to check if the HIP has penetrated or not. With these methods, it is very often that the HIP very quickly penetrates depp into the surface when the 
potential region can not be found and the first collision happens without the detection.

To testify the collision detection, an experiment is performed to use two HIPs controlled by a single haptic device. Fig. 11 illustrates the floor scene captured as a streaming point cloud. In the experiment the user moves the haptic towards the floor as fast as possible to check if there is pop-through phenomenon. It can be seen from Fig. 11 that the previous method has greater chance of pop-through, and our method is more robust. The second experiment is made using the same setup that one haptic control two HIPS, but the haptic movement is controlled by the program. In this experiment, four different speeds are chosen, and 1000 trials are performed for each speed. Fig. 12 shows the pop-though number distribution in 1000 trials. This figure shows our continuous collision detection is more robust than the discrete collision detection.

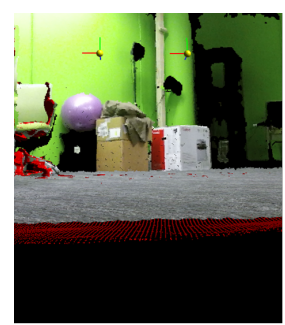

(a)

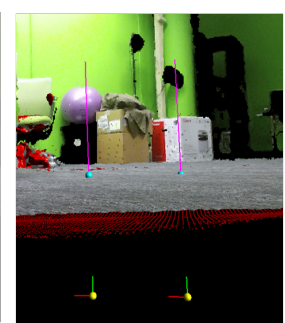

(b)

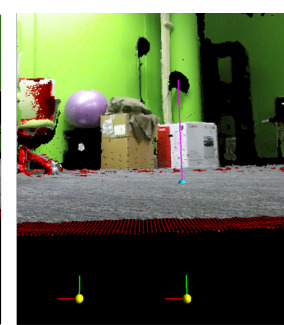

(c)
Figure 11: The comparison results of collision detection between our method and discrete collision detection. (a) There are two copies of the HIP that are controlled by the same haptic device, left HIP uses the discrete collision detection method, right HIP uses our TSDF-based collision detection method. (b) When HIPs are moved at a regular speed, both methods can detect the collision and find proxy. (c) When HIPs are moved at a high speed, only our method can detect the collision.

Since collision detection and proxy finding are usually together to handle, the comparison of efficiency takes both two as one step. In the same environment, discrete collision detection and handling methods such as Leeper's method [14] and Ryden' method [21] take about $1.9 \mathrm{~ms} 3.0 \mathrm{~ms}$. Continuous collision detection and handling methods generally takes a lot more time than discrete methods, however, the time cost of collision detection and handling is about $2.7 \mathrm{~ms}$ on average as shown in Tab. 1 . Therefore, the proposed continuous collision detection has a comparable efficiency comparing with the previous work, and also provides a more robust performance.

\subsection{Proxy Finding}

To validate that the accuracy of proxy finding algorithm, a similar experiment [21] is performed that the HIP is automated on a plane of $500^{*} 500$ point cloud. The HIP is controlled by the program to move towards the opposite direction of plane normal at a constant speed. Fig. 13 shows the y-coordinates of the proxy and the HIP during this procedure. The constant speed of HIP is $0.05 \mathrm{~m} / \mathrm{s}$, the direction is in positive tangential direction to the plane. The proxy
Collision Detection Experiment

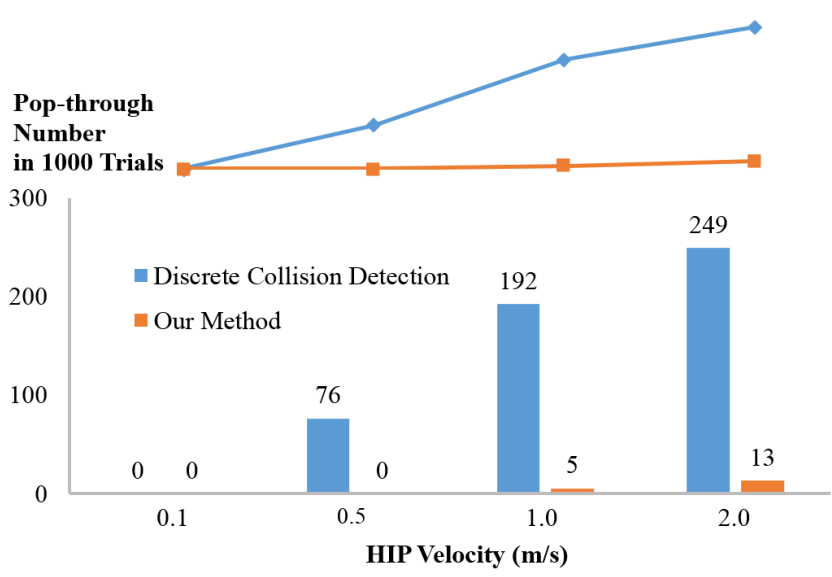

Figure 12: The diagram shows the pop-though number distribution in 1000 trials at different speed. The HIP is controlled to move down automatically towards the floor at a constant speed.

finding is correct since the proxy is always found on the plane and position is stable while moving HIP.

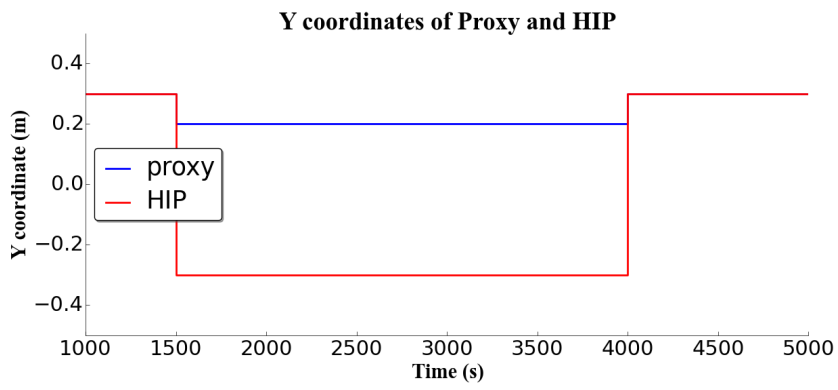

Figure 13: The $Y$ coordinate changes of the proxy and the HIP. At $1,500 \mathrm{~ms}$, the HIP moves under the surface and then after $4,000 \mathrm{~ms}$, it moves on top of surface.

\subsection{Stable Haptic Rendering}

An experiment of haptic Rendering stability is performed, the force curve are shown as Fig. 14. In this experiment, the user is moving the HIP along the Y axis. The force curve demonstrated the stable haptic rendering.

\subsection{Processing Time}

All above experiments are done by using the TSDF resolution $512 * 512 * 512$ inside a volume of $4 \mathrm{~m}^{3}$. The TSDF resolution determines the 3D surface resolution, and further influence the time efficiency of the proposed method. Different TSDF resolutions are chosen to profile the processing time of different component of the proposed method. The results are shown as Tab. 1, for convenience, we have combined some related steps: the collision detection and 


\begin{tabular}{|c|c|c|c|c|c|c|}
\hline \multirow{2}{*}{ TSDF Resolution } & \multicolumn{5}{|c|}{ Average Processing Time } \\
\cline { 2 - 6 } & Total Time & KinectFusion & Surface Update & TSDF Warping & Collision Detection and Handling & HSM \\
\hline $64^{*} 64^{*} 64$ & $14.3 m s$ & $9.7 m s$ & $0.2 m s$ & $0.3 m s$ & $0.6 m s$ & $3.5 m s$ \\
$128^{*} 128^{*} 128$ & $18.4 m s$ & $12.4 m s$ & $0.6 m s$ & $0.8 m s$ & $1.3 m s$ & $3.3 m s$ \\
$256^{*} 256^{*} 256$ & $19.9 m s$ & $13.2 m s$ & $0.8 m s$ & $1.1 m s$ & $2.4 m s$ & $3.2 m s$ \\
$384^{*} 384^{*} 384$ & $29.9 m s$ & $20.5 m s$ & $1.0 m s$ & $2.3 m s$ & $2.7 m s$ & $3.7 m s$ \\
$512^{*} 512^{*} 512$ & $36.6 m s$ & $25.1 m s$ & $1.2 m s$ & $3.8 m s$ & $3.8 m s$ \\
\hline
\end{tabular}

Table 1: Processing time of different components in our method using different TSDF resolution.

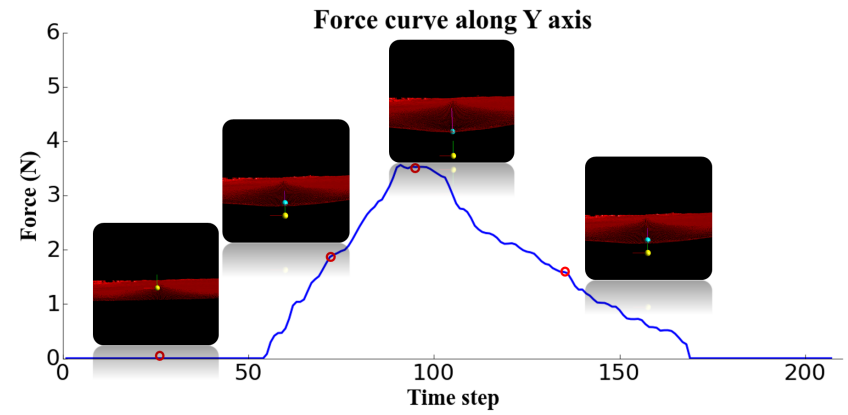

Figure 14: Haptic rendering curve for real-time deformable surface. The user move down and up the HIP along the $Y$ Axis, therefore the force curve only is shown along the $\mathrm{Y}$ direction.

proxy finding are combined as "collision detection and handling". It is observed that all proposed components in our method are very efficient, since most of them are parallelized using GPU. HSM simulation is independent from the other components, therefore its time cost is similar for different TSDF resolution. The proposed method can guarantee the real-time visual rendering, even using a high TSDF resolution. Furthermore, the box-plot of processing time of one iteration is shown in Fig. 15.

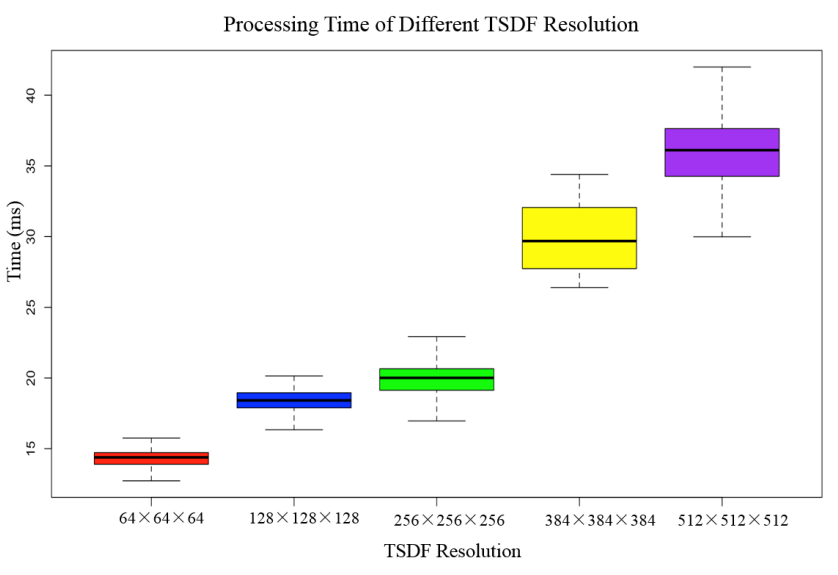

Figure 15: The box-plot of processing time of each iteration using different TSDF resolution. The trial for each resolution lasts for 30 minutes.

\subsection{Tele-immersive Wound Cleaning Training System}

Based on the proposed method, a wound cleaning tele-immersive training system is developed, as shown in the Fig. 1. This system includes the remote site for the physician and local site for the trainee and the patient. At local site the trainee uses Kinect to capture the wound, and a virtual environment with patient's 3D surface is rendered on TV. At remote site the physician can use haptic device to interact with the virtual body to show how to do the wound cleaning. The work flow of the system is as follows: The physician uses the haptic device to mark the location around the wound that needs to be cleaned, and then use haptic device as cloth to show how to clean. After the instructions, the trainee (which may be the patient him/herself) can do the real-world cleaning training. This training system gains more advantages comparing with other tele-immersive systems. First, the real-time physics simulation (deformation) greatly enhance the realistic experience, second, the local wound scanning and remote haptic interaction can be done simultaneously.

\subsection{Experts' Feedback}

The wound cleaning training system is shown to several Physical Medicine and Rehabilitation specialists. They believed the conceptional system could help the remote training and have a big potential for other tele-immersive medical applications. The traditional remote training system for wound care is through the video chat. The specialists think the advantage of our system is providing the 3D surface for haptic interaction. The haptic interaction can generate more realistic experience for physicians, and provide more clear instructions for the trainees (for example the deformation can show the visual cue for the pushing direction). The proposed system also inspired them for more ideas, some physician suggests the idea of using a virtual wound to show patients how to care for their own wound. The various simulations including deformation can show them when to suspect infection, etc.

\section{CONCLUSION AND DISCUSSION}

The proposed method provides a fast and stable haptic rendering for streaming deformable surface. Besides KinectFusion, most of modules are able to be parallelized using GPU, therefore this method can perform efficient haptic interaction together with deformation and fusion. To the best of our knowledge, the proposed method is the first work that exploits the advantages from both KinectFusion and hapic interaction with complex physics simulation. KinectFusion 
provides real-time 3D dense reconstruction of regions of interest, while haptic-enabled deformation enables the user to freely remote touch the streaming surface on the fly. The experiment results demonstrate the smooth deformation, even the deformed surface is being fused. This feature can be applied to lots of potential virtual reality / tele-immersive applications such as tele-immersive medical training and remote designing.

There are many future works that can improve the current system. Currently the haptic rendering of the proposed method is 3 degree of freedom, this method will be naturally extended for 6 degree of freedom haptic rendering in the future. Besides the surface physics simulation, the deformation can be also applied for haptic avatar. The proposed method defines the HSM volumetric meshes with the same size of TSDF volume, the deformation field takes the whole scene together to perform the simulation. However, in most virtual reality / tele-immersive applications, the virtual objects are more flexible if they have different physics parameters. Therefore, in the future, vision algorithms can be applied to segment the objects, and then those objects are assigned with different physics properties. Furthermore, since the proposed method focuses on the semi-static surface, the proposed method cannot be directly used in dynamic scenes or objects. Recent research [16] proposed the real-time method for real-time fusion of the non-rigid scene. It is a more challenging problem to add haptic interaction with such dynamic scene.

\section{ACKNOWLEDGEMENTS}

This material is based upon work supported by the National Science Foundation (NSF) under Grant No. 1012975 and US Army Research Office (ARO) STIR (Short Term Innovative Research) Grant 67369CS. Any opinions, findings, and conclusions or recommendations expressed in this material are those of the author(s) and do not necessarily reflect the views of the NSF and ARO. Chao Li and Xiaohu Guo are partially supported by National Science Foundation (NSF) under Grant No. IIS-1149737.

\section{REFERENCES}

[1] Jernej Barbič and Doug L. James. 2008. Six-DoF Haptic Rendering of Contact Between Geometrically Complex Reduced Deformable Models. EEE Trans. Haptics 1, 1 (Jan. 2008), 39-52.

[2] Robert Bridson, Ronald Fedkiw, and John Anderson. 2002. Robust treatment of collisions, contact and friction for cloth animation. ACM Transactions on Graphics (ToG) 21, 3 (2002), 594-603.

[3] Tyson Brochu, Essex Edwards, and Robert Bridson. 2012. Efficient geometrically exact continuous collision detection. ACM Transactions on Graphics (TOG) 31, 4 (2012), 96.

[4] Jongeun Cha, Mohamad Eid, and Abdulmotaleb El Saddik. 2008. Dibhr: depth image-based haptic rendering. In International Conference on Human Haptic Sensing and Touch Enabled Computer Applications. Springer, 640-650.

[5] Jongeun Cha, Seung-man Kim, Ian Oakley, Jeha Ryu, and Kwan H Lee. 2005. Haptic interaction with depth video media. In Pacific-Rim Conference on Multimedia. Springer, $420-430$.

[6] Sonny Chan, Nikolas H Blevins, and Kenneth Salisbury. 2013. Deformable haptic rendering for volumetric medical image data. In World Haptics Conference (WHC) 2013. IEEE, 73-78.

[7] Christian Duriez, Frederic Dubois, Abderrahmane Kheddar, and Claude Andriot 2006. Realistic haptic rendering of interacting deformable objects in virtual environments. IEEE transactions on visualization and computer graphics 12, 1 (2006), 36-47.

[8] Susan Fisher and Ming C Lin. 2001. Deformed distance fields for simulation of non-penetrating flexible bodies. In Computer Animation and Simulation 2001 Springer, 99-111.

[9] Nico Galoppo, Serhat Tekin, Miguel A Otaduy, Markus Gross, and Ming C Lin. 2007. Interactive haptic rendering of high-resolution deformable objects. In
Virtual Reality. Springer, 215-223.

[10] Xiaohu Guo, Jing Hua, and Hong Qin. 2004. Scalar-function-driven editing on point set surfaces. IEEE Computer Graphics and Applications 24, 4 (2004), 43-52.

[11] Xiaohu Guo, Jing Hua, and Hong Qin. 2004. Touch-based haptics for interactive editing on point set surfaces. IEEE Computer Graphics and Applications 24, 6 (2004), 31-39.

[12] Shahram Izadi, David Kim, Otmar Hilliges, David Molyneaux, Richard Newcombe, Pushmeet Kohli, Jamie Shotton, Steve Hodges, Dustin Freeman, Andrew Davison, and others. 2011. KinectFusion: real-time 3D reconstruction and interaction using a moving depth camera. In Proceedings of the 24th annual ACM symposium on User interface software and technology. ACM, 559-568.

[13] Thomas C Knott and Torsten W Kuhlen. 2016. Accurate and adaptive contact modeling for multi-rate multi-point haptic rendering of static and deformable environments. Computers \& Graphics 57 (2016), 68-80.

[14] Adam Leeper, Sonny Chan, and Kenneth Salisbury. 2012. Point clouds can be represented as implicit surfaces for constraint-based haptic rendering. In Robotics and Automation (ICRA), 2012 IEEE International Conference on. IEEE, 5000-5005.

[15] Matthias Müller, Bruno Heidelberger, Matthias Teschner, and Markus Gross. 2005. Meshless deformations based on shape matching. In ACM Transactions on Graphics (TOG), Vol. 24. ACM, 471-478.

[16] Richard A Newcombe, Dieter Fox, and Steven M Seitz. 2015. Dynamicfusion: Reconstruction and tracking of non-rigid scenes in real-time. In Proceedings of the IEEE conference on computer vision and pattern recognition. 343-352.

[17] Richard A Newcombe, Shahram Izadi, Otmar Hilliges, David Molyneaux, David Kim, Andrew J Davison, Pushmeet Kohi, Jamie Shotton, Steve Hodges, and Andrew Fitzgibbon. 2011. KinectFusion: Real-time dense surface mapping and tracking. In Mixed and augmented reality (ISMAR), 2011 10th IEEE international symposium on. IEEE, 127-136.

[18] M.A. Otaduy and M.C. Lin. 2005. Stable and responsive six-degree-of-freedom haptic manipulation using implicit integration. In Eurohaptics Conference, 2005 and Symposium on Haptic Interfaces for Virtual Environment and Teleoperator Systems, 2005. World Haptics 2005. First foint. 247-256.

[19] Shahzad Rasool and Alexei Sourin. 2016. Real-time haptic interaction with RGBD video streams. The Visual Computer (2016), 1-11.

[20] Diego C Ruspini, Krasimir Kolarov, and Oussama Khatib. 1997. The haptic display of complex graphical environments. In Proceedings of the 24th annual conference on Computer graphics and interactive techniques. ACM Press/Addison-Wesley Publishing Co., 345-352.

[21] Fredrik Ryden and Howard Jay Chizeck. 2013. A proxy method for real-time 3-DOF haptic rendering of streaming point cloud data. IEEE transactions on Haptics 6, 3 (2013), 257-267.

[22] Kenneth Salisbury and Christopher Tarr. 1997. Haptic rendering of surfaces defined by implicit functions. In ASME Dynamic Systems and Control Division, Vol. 61. 61-67.

[23] Min Tang, Sean Curtis, Sung-Eui Yoon, and Dinesh Manocha. 2009. ICCD: Interactive continuous collision detection between deformable models using connectivity-based culling. IEEE Transactions on Visualization and Computer Graphics 15, 4 (2009), 544-557.

[24] Yuan Tian, Yin Yang, Xiaohu Guo, and B. Prabhakaran. 2013. Haptic-Enabled Interactive Rendering of Deformable Objects based on Shape Matching (IEEE International Symposium on Haptic Audio Visual Environments and Games). 7580 .

[25] Yuan Tian, Yin Yang, Xiaohu Guo, and Balakrishnan Prabhakaran. 2015. Stable haptic interaction based on adaptive hierarchical shape matching. Computational Visual Media 1, 3 (2015), 253-265.

[26] Rodolphe Vaillant, Loïc Barthe, Gaël Guennebaud, Marie-Paule Cani, Damien Rohmer, Brian Wyvill, Olivier Gourmel, and Mathias Paulin. 2013. Implicit skinning: real-time skin deformation with contact modeling. ACM Transactions on Graphics (TOG) 32, 4 (2013), 125.

[27] Hongyi Xu and Jernej Barbic. 2016. 6-DoF Haptic Rendering using Continuous Collision Detection Between Points and Signed Distance Fields. IEEE Transactions on Haptics (2016). 